\title{
PREVENÇÃO E CONTROLE DE INFECÇÃO EM UNIDADE DE TERAPIA INTENSIVA NEONATAL
}

\author{
Elisiane LORENZINI ${ }^{\mathrm{a}}$, Tatiane Costa da $\operatorname{COSTA}^{\mathrm{b}}$, Eveline Franco da SILVA $^{\mathrm{c}}$
}

\section{RESUMO}

Objetivou-se identificar o conhecimento da equipe de enfermagem de uma Unidade de Terapia Intensiva Neonatal (UTIN) sobre o controle de infecção, identificando os fatores que facilitam ou dificultam o controle e prevenção das Infecções Relacionadas à Assistência a Saúde (IRAS). Estudo descritivo com abordagem qualitativa, realizado com três enfermeiras e 15 técnicas de enfermagem, que atuam em uma UTIN de uma instituição filantrópica, na região sul do Brasil. Evidenciou-se que a equipe de enfermagem possui grande conhecimento sobre os fatores que facilitam a prevenção e controle das IRAS em UTIN, sendo o principal, a higienização das mãos. Entre os fatores que dificultam o controle e prevenção, estão a superlotação e a excessiva carga de trabalho. A atuação eficiente e qualificada da equipe de enfermagem constitui-se em estratégia de prevenção e controle das IRAS.

Descritores: Controle de infecções. Infecção hospitalar. Enfermagem neonatal. Unidades de terapia intensiva neonatal.

\section{RESUMEN}

Se objetivó identificar los conocimientos del equipo de enfermería de una Unidad de Terapia Intensiva Neonatal (UTIN), sobre el control de infección, identificando los factores que facilitan o dificultan el control y prevención de las Infecciones Relacionadas a la Atención a la Salud (IRAS). Estudio con abordaje cualitativo, realizado con tres enfermeras y quince técnicas de enfermería, que actúan en una UTIN de una institución filantrópica, en la región sur de Brasil. Se ha evidenciado que el equipo de enfermería tiene gran conocimiento sobre los factores que facilitan la prevención y control de las IRAS en UTIN, siendo la principal, higienización de las manos. Entre los factores que dificultan el control y prevención están el hacinamiento y la excesiva carga de trabajo. La actuación eficiente y cualificada del equipo de enfermería se constituye de estrategias de prevención y control de las IRAS.

Descriptores: Control de infecciones. Infección hospitalaria. Enfermería neonatal. Unidades de cuidado intensivo neonatal. Título: Prevención y control de la infección en la unidad de cuidados intensivos neonatal.

\section{ABSTRACT}

This study was aimed to identify the knowledge of the nursing team of a Neonatal Intensive Care Unit (NICU) on infection control, identifying the factors that facilitate or hinder the prevention and control of Healthcare Associated Infections (HCAI). A descriptive study using a qualitative research method conducted with three nurses and 15 nurse technicians, who work in a NICU of a charitable organization, in southern Brazil. It became evident that the nursing staff had great knowledge about the factors that facilitate the prevention and control of HCAI in NICU, the most important factor being proper hand hygiene. Among the factors that hinder infection prevention and control are to overcrowding and excessive workload. The efficient performance of the nursing staff is an important part of the strategy for prevention and control of HCAI.

Descriptors: Infection control. Cross infection. Neonatal nursing. Intensive care units, neonatal.

Title: Infection prevention and control in neonatal intensive care unit.

\footnotetext{
a Enfermeira, Especialista em Gerenciamento em Enfermagem, Mestre em Ciências da Saúde, Docente da Faculdade Nossa Senhora de Fátima/ FÁTIMA, Caxias do Sul e da Universidade FEEVALE, Novo Hamburgo (RS), Brasil.

b Enfermeira, Faculdade Nossa Senhora de Fátima. Caxias do Sul (RS), Brasil.

c Enfermeira Obstetra, Especialista em Saúde Pública, Mestranda, Programa de Pós-Graduação em Enfermagem, Universidade Federal do Rio Grande do Sul/PPGENF/UFRGS. Docente da Faculdade Nossa Senhora de Fátima. Caxias do Sul (RS), Brasil.
} 


\section{INTRODUÇÃO}

As Infecções Relacionadas à Assistência à Saúde (IRAS) representam um grande problema para a segurança e qualidade de vida do paciente, além disso, seu impacto pode resultar em morte, hospitalização prolongada, incapacidade ao longo prazo, um grande encargo financeiro às instituições de saúde e custo elevado para o paciente e seus familiares ${ }^{(1)}$.

Devido à alta incidência de IRAS, no Brasil, estima-se que $3 \%$ a $15 \%$ dos pacientes hospitalizados desenvolvem Infecção Hospitalar (IH) $)^{(2)}$, o Ministério da Saúde criou o Programa de Controle de Infecções Hospitalares (PCIH), que é um conjunto de ações desenvolvidas deliberada e sistematicamente, com o objetivo à redução máxima da incidência dessas infecções. A Portaria nº 2.616/98 regulamenta o Programa Nacional de Controle de Infecção e a implantação da Comissão de Controle de Infecção Hospitalar (CCIH). Dentre diversas atribuições do CCIH está a educação permanente com o objetivo de prevenção e controle dessas IRAS ${ }^{(3)}$.

Apesar de todos os esforços da OMS e do Ministério da Saúde, recente publicação em periódico de abrangência internacional revela que dentre os diversos desafios que persistem na área da saúde, no Brasil, está o de reduzir a incidência de infecções adquiridas nos serviços de saúde. Os autores consideram que esse seja um problema de grande magnitude, pois muitos hospitais contam com $\mathrm{PCIH}$ mal estruturados e não priorizam a vigilância ${ }^{(4)}$. Há também evidências de que essas infecções são uma das principais causas de morbimortalidade neonatal nos países em desenvolvimento ${ }^{(5)}$.

As IRAS nas Unidades de Terapia Intensiva Neonatal (UTIN) são aquelas adquiridas no período intraparto (origem materna e manifesta em 48 horas de vida), durante a hospitalização, ou 48 horas após a alta, com exceção das infecções transplacentárias ${ }^{(6)}$. O cuidado com o recém-nascido deve ser diferenciado e minucioso, pois sua pele é a principal porta de entradas dessas infecções ${ }^{(7)}$. Portanto, a equipe de enfermagem das unidades de tratamento intensivo neonatal e a CCIH devem trabalhar em conjunto, para detectar possíveis falhas a fim de melhorar a qualidade de vida do $\mathrm{RN}^{(8)}$.

Existem diversos fatores de riscos em uma UTIN, dentre os quais se destacam: a realização de procedimentos invasivos, o tempo de internação, baixo peso ao nascimento, o contato precoce com os pais, todos estes fatores podem desencadear uma proliferação maior das IRAS, prejudicando a recuperação e qualidade de vida do $\mathrm{RN}^{(9)}$.

A motivação para a escolha desse tema emergiu ao assistir as aulas da disciplina de Controle de Infecção nos Serviços de Saúde, durante o curso de graduação em enfermagem, de uma das autoras do trabalho. Após muitas pesquisas em bases de dados eletrônicas verificou-se que, mesmo sendo um tema de muita relevância nas instituições de saúde, poucos estudos são realizados sobre prevenção e controle de IRAS em UTIN. Neste contexto, o problema da pesquisa pauta-se nas dúvidas sobre o conhecimento dos profissionais sobre os riscos de infecção no intensivismo neonatal e a forma de preveni-las.

Diante de tais considerações, objetivou-se neste estudo identificar o conhecimento da equipe de enfermagem de uma Unidade de Terapia Intensiva Neonatal (UTIN), sobre o controle de infecção, identificando os fatores que facilitam ou dificultam o controle e prevenção das Infecções Relacionadas à Assistência a Saúde (IRAS).

\section{MÉTODO}

Trata-se de um estudo de cunho descritivo com abordagem qualitativa e técnica de análise de conteúdo temática. Optou-se por esta abordagem por implicar no entendimento/interpretação dos sentidos e das significações que uma pessoa dá aos fenômenos em foco ${ }^{(10)}$.

A investigação ocorreu na UTI neonatal de uma instituição filantrópica localizada em um município da região sul do Brasil. Participaram desta pesquisa 18 integrantes da equipe de enfermagem (três enfermeiras e 15 técnicas de enfermagem). O critério de inclusão estabelecido foi: ser colaborador da UTI neonatal com mais de três meses de contratação. Excluíram-se as enfermeiras que ocupavam cargos de chefia. A determinação do número de participantes baseou-se no critério de saturação de dados, definido quando as informações coletadas passam a apresentar uma quantidade de repetições em seu conteúdo, ou seja, o pesquisador entende que novas falas passam a ter acréscimos pouco significativos em vista dos objetivos inicialmente propostos para a pesquisa ${ }^{(10)}$.

A coleta de dados ocorreu durante o mês de setembro de 2012, por meio de um roteiro de 
entrevista semiestruturada, elaborado pelas pesquisadoras, composto por 11 perguntas que enfocaram o controle de infecção em UTI neonatal. As entrevistas foram agendadas com os participantes e realizadas individualmente nas instalações da referida instituição. Com autorização dos participantes, as entrevistas foram gravadas em gravador digital e posteriormente transcritas para melhor aproveitamento dos dados.

O tratamento dos dados fundamentou-se na técnica de análise de conteúdo temática, que tem por objetivo obter por procedimentos sistemáticos e objetivos de descrições do conteúdo das mensagens. Esta análise segue três etapas: a pré-análise, na qual foi realizada a organização dos materiais, com o objetivo de tornar as idéias iniciais esquemáticas e operacionais, para isso foi realizada uma leitura exaustiva e repetida dos dados transcritos das gravações; a exploração do material, que é a administração sistemática das decisões anteriormente tomadas, que é a seleção das falas das entrevistadas e organização das categorias; e na terceira etapa foi realizado o tratamento dos resultados com sua interpretação ${ }^{(11)}$. As categorias temáticas elencadas a priori foram: fatores que contribuem para a prevenção e controle das IRAS na UTIN; e fatores que dificultam a prevenção e controle das IRAS na UTIN.

A pesquisa respeitou as diretrizes da Resolução 196/96 do Conselho Nacional de Saúde, foi autorizada pela instituição cenário do estudo e aprovada pelo Comitê de Ética em Pesquisa da instituição de origem do estudo, com parecer número 82.629/2012. Para garantia do anonimato os participantes da pesquisa foram identificados pela letra $\mathrm{E}$, de entrevistado, seguida de um número conforme ordem cronológica da realização das entrevistas (E01, EO2,..., E18).

\section{RESULTADOS E DISCUSSÃO}

Na caracterização dos participantes verificou-se que todos eram do sexo feminino, quanto à idade, variou entre 20 anos e 43 anos. Oito das participantes tinham de um a cinco anos de formação, oito, entre seis e dez anos de formação e duas tinham mais de dez anos de formação. Duas das três enfermeiras entrevistadas possuíam curso de pós-graduação lato sensu (neonatologia e urgência e emergência pediátrica e adulto).

\section{Fatores que contribuem para a prevenção e controle das IRAS na UTIN}

As participantes demonstraram em suas falas que detêm conhecimento sobre a prevenção e controle das IRAS na UTIN. Ao relatarem os principais métodos de controle e prevenção evidenciou-se esse conhecimento:

Lavagem de mãos, o uso de álcool gel, luvas, assepsia na hora da medicação [...]. (EO1)

Eu acho que o principal é a lavagem de mãos, uma boa lavagem de mãos. (E14)

A lavagem das mãos, o uso do álcool gel, as luvas, as limpezas também das incubadoras. (E18)

Nesses depoimentos as entrevistadas demonstraram a amplitude desse conhecimento, pois acreditam que a higienização das mãos (HM) é o principal método para prevenir e controlar as IRAS na UTIN. Estudos comprovam que a melhor forma de prevenir as infecções em ambiente hospitalar é a correta HM antes e após a manipulação dos pacientes $^{(6,12)}$. Este procedimento é reconhecido como o mais importante e menos dispendioso para evitar a transmissão das $\operatorname{IRAS}^{(12)}$. A melhor maneira de interromper a transmissão antimicrobiana de organismos resistentes é a correta maneira de HM, diminuindo as IRAS por contato com pacientes, podendo contribuir para a diminuição da incidência de bactérias resistentes ${ }^{(13)}$.

No entanto, apesar das evidências e da disponibilidade de produtos e técnicas para realizar adequadamente a HM, muitos profissionais de saúde o fazem de maneira não satisfatória, sem observar as recomendações, não realizando a HM em aproximadamente $60 \%$ das vezes que seria necessário ou indicado ${ }^{(14)}$. Outros achados corroboram essas evidências, relatando que os profissionais da área da saúde possuem conhecimentos sobre a técnica correta da HM e reconhecem a importância desta medida na prevenção de infecções cruzadas, no entanto, higienizam suas mãos de maneira incorreta $^{(6,7)}$. Portanto, embora a HM tenha um grande valor epidemiológico na prevenção das IRAS, a adesão a esta medida tem sido um dos maiores desafios para o CCIH, visto que as IRAS estão associadas à baixa adesão dos profissionais da área da saúde quanto à $\mathrm{HM}^{(15)}$. 
O uso de álcool gel para a HM e o uso de álcool $70 \%$ para a higienização dos materiais, também foram mencionados pelos participantes deste estudo. A maneira mais efetiva e segura de desinfecção de materiais e superfícies é o uso do álcool $70 \%{ }^{(16)}$.

Além da HM, as participantes do estudo reconhecem outros métodos que contribuem para a prevenção e controle das IRAS na UTI neonatal, como o uso controlado de antimicrobianos:

[...] não utilizar antibióticos sem precisar [...]. (E06)

Embora o uso de antimicrobianos seja recomendado apenas com prescrição médica, a enfermagem está atenta e ciente que o uso indiscriminado pode acarretar vários prejuízos à saúde do recém-nascido, visto que o uso excessivo de antimicrobianos é fator predisponente no desencadear de possíveis IRAS, uma vez que a resistência bacteriana em um ser com o sistema imunológico totalmente comprometido pode ser potencialmente fatal. Então, deve-se garantir o uso racional desses medicamentos impedindo assim, a propagação da resistência antimicrobiana ${ }^{(17)}$.

As participantes também salientaram a importância de evitar a contaminação cruzada, expresso nas falas:

[...] se tiver dentro de uma sala tipo aqui no isolamento daí aquela [técnica] que está no isolamento procura não sair pra outras salas, não ficar podendo contagiar outras salas [...] quem está com o bebê do isolamento fica só com o bebê, não mexe em outro. (E17)

[...] sempre estar atento no procedimento que tu vai fazer para não contaminar, evitar manusear em paciente, depois mexer em outro é muito importante, manusear só quando necessário. (EO9)

[...] porque acaba tocando um paciente, mexe em um, toca no outro, então assim tem que lavar as mãos sempre, antes e depois de mexer no bebê [...]. (EO3)

Sabe-se que as mãos dos profissionais são o principal meio de transporte dessas infecções. A pele do RN é a principal porta de entrada de microrganismos patógenos, sendo que cerca de $80 \%$ dos recém-nascidos prematuramente desenvolvem alguma injúria na pele até o primeiro mês de vida e aproximadamente $25 \%$ de todos os pré-termos e de baixo peso, terão ao menos um episódio de sepse até o terceiro dia de vida ${ }^{(5,7)}$.
A orientação aos pais quanto ao manuseio do seu recém-nascido, foi citada como importante fator que contribui para a prevenção das IRAS, conforme expressa o fragmento da fala de E10:

[…] os cuidados com os pais, tem muitos pais que vem da rua, o uso do jaleco nos pais teria que ter, tem muitos pais que não usam [...] mas sempre é bom um cuidado a mais sempre é bom. (E1O)

A educação em saúde, por meio de orientações, constitui-se em importante estratégia de prevenção de infecções, no entanto, um dos grandes obstáculos enfrentados pelos profissionais na área da saúde é o não entendimento dos familiares sobre as fontes de risco. A presença dos pais junto ao RN é muito importante, visto que, o mesmo necessita de todo o carinho e conforto para sua recuperação e estabilidade, além disso, os pais se sentem excluídos sem estes gestos, mas para prevenir e controlar as IRAS não basta apenas permitir e incentivar a permanência dos pais na UTIN é necessário orientar as medidas preventivas de infecções, bem como a maneira correta de manusear seu bebê, tocando-o com o máximo de cuidado possível, ensinando assim a preveni-las e controlá-las na UTIN. Sabe-se que as infecções têm origem em vários fatores, tais como a condição do paciente, a severidade da doença, o fluxo de entrada de visitas de familiares a estes pacientes e o tempo de internação. No entanto, ressalta-se que se não houver certo controle por parte da equipe de enfermagem ocorrerá uma tendência maior à proliferação de $\operatorname{IRAS}^{(18)}$.

\section{Fatores que dificultam a prevenção e controle das IRAS na UTIN}

A superlotação nas unidades de internação neonatais é frequente em todo o país. Isso tem um importante impacto nas IRAS, pois dificultam a qualidade do atendimento assistencial ao RN. Esse aspecto foi relatado por algumas das participantes, conforme é expresso nas falas de E07 e E16:

Superlotação [...], mas a gente tem crianças graves em fim e que em algum momento de repente se passa, né? Uma intercorrência a gente lava a mão, mas não passa o álcool, ou não calça a luva. [...]. (EO7)

[...] alguma coisa assim, a superlotação de pacientes, fica muito difícil, quando tem um grande número de bebês. (E16) 
A superlotação nos serviços de saúde é um problema nacional que representa um forte fator percebido pelos profissionais de saúde e que dificulta a prevenção e controle das IRAS. Nesta pesquisa, os depoimentos dos participantes vão ao encontro de um estudo realizado com 12 integrantes da equipe multiprofissional de uma UTIN da cidade do Rio de Janeiro, no Brasil, na qual os entrevistados também relataram como grande problema a superlotação, o que gera dificuldades na prestação do cuidado e da assistência humanizada ${ }^{(19)}$.

A UTIN é um lugar diferenciado das demais áreas hospitalares, pois os recém-nascidos necessitam de um cuidado especializado e minucioso, além disso, as intercorrências são frequentes nesta unidade e foram citadas pelas participantes como um dos fatores que dificultam a prevenção das IRAS na UTIN:

[...] às vezes acontece tipo em uma intercorrência, o nenê parou tu não tem muito, por mais que tu tente fazer não vai ser a mesma higienização de uma ampola que tu faz com calma quando está preparando uma medicação [… (EO3)

[...] fica mais difícil às vezes quando se trabalha com um número maior de pacientes na hora de uma intercorrência porque às vezes é tudo muito rápido e fica difícil mesmo fazer tudo: lavar mão daí às vezes tem coisa que um segundo já faz diferença, e eu acho que é isso que dificulta mais […]. (E11)

Sabe-se que a atuação da equipe de enfermagem durante as intercorrências em UTI neonatal exige muita habilidade técnica, conhecimentos específicos e atualizados e agilidade ${ }^{(19)}$, porém nestes eventos muitas vezes os profissionais não realizam a HM de forma adequada, ou não usam equipamentos de proteção individual como deveriam, o que pode acarretar em infecções. Portanto, as intercorrências, quando não são bem gerenciadas, podem configurar-se como um fator que contribui no aumento das IRAS.

Outro fator destacado pelas entrevistadas é a sobrecarga de trabalho juntamente com a redução do quadro funcional e o excesso de pacientes destinados a cada membro da equipe de enfermagem, para a execução dos cuidados, conforme se apresenta nas falas de E06 e E17:

Pouco pessoal, excesso de pacientes, falta de informação, sobrecarga de trabalho. (EO6)
Dificultam quando tem assim, quando a gente está com dois, precisa ficar com dois bebês e um está em isolamento e o outro não, dai e não tem funcionário [...]. (E17)

O tema carga de trabalho é discutido mundialmente e é muito importante no atendimento de enfermagem em UTIN devido ao surgimento de novas tecnologias no cuidado, bem como a necessidade de um cuidado especial a pacientes gravemente enfermos e a necessidade de mão de obra especializada. Em um estudo prospectivo de coorte, envolvendo 543 recém-nascidos, que abordou a relação entre a carga de trabalho e os eventos adversos (EAs) relacionados à ventilação mecânica em duas UTIN no Brasil, revelou uma taxa crescente de eventos, quando a demanda de cuidados dos enfermeiros e auxiliares eram altas. Com número de recém-nascidos classificados de acordo com a demanda de cuidados (RCDCs) de 3,8 por auxiliar de enfermagem, as taxas de EAs de ventilação mecânica dobraram, e com RCDCs de 4,8 as taxas triplicaram. Os autores demonstraram associação entre carga de trabalho dos profissionais e as taxas de EAs, indicando conexão causal entre carga de trabalho e segurança do paciente ${ }^{(20)}$.

Nesse contexto, o dimensionamento de pessoal de enfermagem em uma UTIN, além da melhoria do cuidado assistencial prestado aos pacientes, contribui para que sejam fornecidas condições favoráveis de trabalho e, consequentemente, visando à saúde da equipe de enfermagem, que lida diretamente com situações estressante como o sofrimento e a morte, pois na UTIN estão alocados pacientes extremamente debilitados e que necessitam de cuidados intensos e constantes, comumente maiores do que em outras unidades hospitalares.

Outro fator, citado pelas participantes, que dificulta a prevenção e controle das IRAS na UTIN é o uso de adornos, joias e celulares, pois os mesmos são meios de transporte dos microorganismos para os RN:

[...] entra muito acadêmico, normalmente mais os de medicina que tu percebe que eles não fazem a lavagem correta das mãos, usam relógio que aqui não pode, não pode usar relógio, celulares, não pode usar anéis, só aliança, eu acho que isso tu vê bastante o pessoal da medicina que não respeita isso. (EO4)

A ANVISA orienta que antes de iniciar qualquer uma das técnicas com RN é necessário 
retirar todo e qualquer adorno, como, joias, anéis, aliança, pulseiras e relógio, pois sob tais objetos podem acumular-se microrganismos ${ }^{(12)}$. Denota-se, portanto, que os profissionais da saúde são agentes importantes e determinantes para prevenção e controle das IRAS na UTIN, pois possuem grande influência no cuidado com o RN, cujo objetivo é melhorar a qualidade de vida dos mesmos.

\section{CONSIDERAÇÕES FINAIS}

Os resultados deste estudo demonstraram que a equipe de enfermagem conhece os fatores que contribuem e aqueles que dificultam a prevenção e controle de IRAS em UTIN. Nesse sentido, constitui-se em avanço no conhecimento acerca do tema, pois possibilita o planejamento de estratégias específicas, de educação permanente, a serem utilizadas na abordagem de prevenção de IRAS.

A correta HM foi mencionada por todos os participantes do estudo, e atualmente é o método mais eficaz e menos dispendioso na prevenção e controle das IRAS. A excessiva carga de trabalho da equipe, o uso de adornos, as intercorrências e a superlotação das unidades foram citados como fatores que dificultam a prevenção e controle das IRAS, corroborando com dados prévios descritos na literatura.

Acredita-se que a atuação eficiente e qualificada da equipe de enfermagem, constitui-se em estratégia de prevenção e controle das IRAS e contribui de forma pró-ativa para melhorar a qualidade de vida do recém-nascido. Cabe salientar que a prevenção e controle das IRAS devem ser realizados por todos os membros da equipe multidisciplinar, atuando em conjunto para o bem estar do recém-nascido.

As limitações deste estudo relacionam-se ao fato de se ter entrevistado apenas profissionais de uma instituição hospitalar. No entanto, defende-se que tais limitações não comprometeram os resultados, visto que se trata de uma abordagem qualitativa sobre o risco de infecção em unidade de intensivismo neonatal, na qual não se pretende generalizar os fenômenos. Contudo, sugere-se que sejam realizados outros estudos sobre a temática, de diferentes métodos, em diferentes instituições, para que se possam criar novas estratégias de cuidado de enfermagem que contribuam para a prevenção e controle de infecções em unidades de terapia intensiva neonatal.

\section{REFERÊNCIAS}

1 World Health Organization (WHO). Report on the burden of endemic health care-associated infection worldwide - clean care is safer care. Geneva: WHO; 2011.

2 Conselho Regional de Medicina (São Paulo). O controle da infecção hospitalar no estado de São Paulo [Internet]. São Paulo; 2010 [citado 2013 Set 01]. Disponível em: http://www.mp.sp.gov.br/portal/page/ portal/Saude_Publica/infeccao_hospitalar_2010.pdf

3 Ministério da Saúde (BR). Portaria n. 2.616, de 12 de maio de 1998 [Internet]. Brasília (DF): 1998 [citado 2013 Set 01]. Disponível em: http:// bvsms.saude.gov.br/bvs/saudelegis../gm/1998/ prt2616_12_05_1998.html.

4 Victora CG, Barreto ML, Leal MC, Monteiro CA, Schmidt MI, Paim J, et al. Condições de saúde e inovações nas políticas de saúde no Brasil: o caminho a percorrer. The Lancet. 2011;90-102. (Série Saúde no Brasil 6).

5 Ganatra HA, Zaide AKM. Neonatal infections in the developing world. Semin Perinatol. 2010;34(6):416-25.

6 Martinez MR, Campos LAAF, Nogueira PCK. Adesão à técnica de lavagem de mãos em unidade de terapia intensiva neonatal. Rev Paul Pediatr. 2009;27(2):179-85.

7 Adriano LSM, Freire ILS, Pinto JTJM. Cuidados intensivos com a pele do recém-nascido pré-termo. Rev Eletr Enferm [Internet]. 2009 [citado 2012 Nov 02];11(1):173-80. Disponível em: http://www. fen.ufg.br/fen_revista/v11/n1/pdf/v11n1a22.pdf.

8 Montanholi LL, Merighi MAB, Jesus MCP. The role of the nurse in the neonatal intensive care unit: between the ideal, the real and the possible. Rev Latinoam Enferm. 2011;19(2):301-8.

9 Pinheiro MSB, Nicoletti C, Boszczowsk I, Puccini DMT, Ramos SRTS. Infecção hospitalar em unidade de terapia intensiva neonatal: há influência do local do nascimento. Rev Paul Pediatr. 2009;27(1):6-14.

10 Turato ER. Tratado da metodologia da pesquisa clínico-qualitativa: construção teórico-epistemológica, discussão comparada e aplicação nas áreas da saúde e humanas. $5^{\text {a }}$ ed. Petrópolis: Vozes; 2011.

11 Bardin L. Análise de conteúdo. $4^{\mathrm{a}}$ ed. Lisboa: Edições 70; 2009. 
12 Agência Nacional de Vigilância Sanitária (BR). Segurança do paciente: relatório sobre autoavaliação para higiene das mãos. Brasília (DF): ANVISA; 2012.

13 Locks L, Lacerda JT, Gomes ET, Serra ACP. Qualidade da higienização das mãos de profissionais atuantes em unidades básicas de saúde. Rev Gaucha Enferm. 2011;32(3):569-75.

14 Locks L, Lacerda JT, Gomes E, Serratine ACP. Qualidade da higienização das mãos de profissionais atuantes em unidades básicas de saúde. Rev Gaucha Enferm. 2011;32(3):569-75.

15 Kampf G, Löffler H, Gastmeier P. Hand hygiene for the prevention of nosocomial infections. Dtsch Arztebl Int. 2009;106(40):649-55.

16 Oliveira T, Canettieri ACV. Eficiência dos métodos microbiológicos e de ATP-bioluminescência na de- tecção da contaminação de diferentes superfícies. Rev Inst Adolfo Lutz. 2010;69(4):467-74.

17 Thaver D, Ali AS, Zaidi AK. M. Antimicrobial resistance among neonatal pathogens in developing countries. Pediatr Infect Dis J. 2009;28(1 supl):S19-21.

18 Costa R, Padilha MI. Percepção da equipe de saúde sobre a família na UTI neonatal: resistência aos novos saberes. Rev Enferm UERJ. 2011;19(2):231-5.

19 Souza KMO, Ferreira SD. Assistência humanizada em UTI neonatal: os sentidos e as limitações identificadas pelos profissionais de saúde. Ciênc Saúde Coletiva. 2010;15(2):471-80.

20 Lamy Filho F, Silva AA, Lopes JM, Lamy ZC, Simões VM, Santos AM. Staff workload and adverse events during mechanical ventilation in neonatal intensive care units. J Pediatr (Rio J). 2011;87(6):487-92.

\author{
Endereço do autor / Dirección del autor / \\ Author's address \\ Elisiane Lorenzini \\ Rua Felipe dos Santos, 77, ap. 201 A, Padre Reus \\ 93020-180, São Leopoldo, RS \\ E-mail: elisilorenzini@gmail.com
}

Recebido em: 17.01.2013

Aprovado em: 05.11.2013 